\title{
Constituintes químicos isolados dos galhos e cascas de amapazeiro (Parahancornia amapa, Apocynaceae)
}

\author{
Marycleuma Campos HENRIQUE ${ }^{1 *}$, Rita de Cássia Saraiva NUNOMURA ${ }^{1}$, \\ Sergio Massayoshi NUNOMURA², Suniá Gomes SILVA ${ }^{1}$ \\ 1 Universidade Federal do Amazonas, Instituto de Ciências Exatas - Departamento de Química, Av. Gal. Rodrigo Octávio Jordão Ramos, 6.200 - Japiim, 69079-000, \\ Manaus-Amazonas, Brasil. \\ ${ }^{2}$ Instituto Nacional de Pesquisas da Amazônia, Coordenação de Pesquisas em Produtos Naturais, Av. André Araújo, 2.936 - Petrópolis - CEP $69067-375$ - Manaus -AM, Brasil. \\ * Autor correspondente: marycleuma@yahoo.com.br
}

\section{RESUMO}

Parahancornia amapa (Apocynaceae) é típica da região amazônica e conhecida popularmente como amapazeiro e muito utilizada na medicina popular da região Amazônica. O objetivo desse trabalho foi o estudo fitoquímico dos galhos e cascas dessa espécie. Do extrato diclorometânico dos galhos foram isolados o $\beta$-sitosterol, estigmasterol, além de triterpenóides pentacíclicos, $\alpha$-amirina, $\beta$-amirina, lupeol e friedelina. Do extrato metanólico das cascas foi isolado o alcaloide indólico isositsiriquina. As estruturas desses compostos foram identificadas por análise dos espectros de massa de alta resolução, Ressonância Magnética Nuclear de ${ }^{1} \mathrm{H} \mathrm{e}^{13} \mathrm{C}$ e comparaçóes com dados da literatura. Esse é o primeiro relato de isolamento de alcaloide neste gênero.

PALAVRAS-CHAVE: Triterpenos, alcaloide, esteroides.

\section{Chemical constituents isolated from twigs and barks of amapazeiro (Parahancornia amapa, Apocynaceae)}

\begin{abstract}
Parahancornia amapa (Apocynaceae) is typical of the Amazon region and popularly known as amapazeiro and widely used in folk medicine in the Amazon region. The objective of this work was to study the phytochemical composition of twigs and barks of this species. From the dichloromethane extract of twigs were isolated $\beta$-sitosterol, stigmasterol, and pentacyclic triterpenoids $\alpha$-amyrin, $\beta$-amyrin, lupeol and friedelin. From the methanol extract of barks was isolated indole alkaloid isositsiriquina. The structures of these compounds were identified by analysis of the mass spectra high-resolution, ${ }^{1} \mathrm{H}$ and ${ }^{13} \mathrm{C}$ NMR and comparisons with literature data. This is the first report of isolation of alkaloid in this genus.
\end{abstract}

KEYWORDS: Triterpenes, alkaloid, steroids. 
Parahancornia amapa (Huber) é uma espécie da família Apocynaceae, típica da Amazônia brasileira, especialmente, do estado do Amapá, conhecida popularmente como amapa ou amapazeiro e as cascas e o látex são utilizados medicinalmente pelos nativos como tônico, anti-sífilis (Sobrinho et al. 1991) e repelente (Mendonça et al. 2010). O estudo fitoquímico realizado por Sobrinho et al. (1991) relatam o isolamento dos triterpenos friedelina, $\alpha$-amirina, $\beta$-amirina, lupeol e seus derivados acetilados, quatro ésteres de $3 \beta$-O-acil lupeol, dois $3 \beta-O-3$ '-hidroxiacil lupeol, dois $3 \beta-O-3$ '5'- diidroxiacil lupeol das cascas e látex. Carvalho et al. (2001) relatam em seu estudo com as raízes o isolamento de nove novos $3 \beta$-O-acillupeois, além dos já descritos na literatura para esta espécie. O presente trabalho teve como objetivo o estudo fitoquímico de P. amapa na busca de novas classes de substâncias, além das classes químicas já descritas para o gênero. O estudo teve como resultado isolamento e caracterização estrutural dos esteroides $\beta$-sitosterol e estigmasterol e dos triterpenóides pentacíclicos, $\alpha$-amirina, $\beta$-amirina, lupeol e friedelina. Também contribui com o isolamento do alcaloide isositsiriquina (amostra sete). Esse é o primeiro relato de isolamento dessa classe química para o gênero Parahancornia.

Os espectros de Ressonância Magnética Nuclear (RMN) de ${ }^{1} \mathrm{H} \mathrm{e}{ }^{13} \mathrm{C}$, uni e bidimensionais foram registrados em espectrômetro (Unity Inova, Varian, Palo Alto, CA, USA) operando na frequência de $500 \mathrm{MHz}$ para hidrogênio $\left({ }^{1} \mathrm{H}\right)$ e $125 \mathrm{MHz}$ para carbono- $13\left({ }^{13} \mathrm{C}\right)$. Foram utilizados os solventes $\mathrm{CD}_{3} \mathrm{OD}$ para a amostra sete e o $\mathrm{CDCl}_{3}$ para as demais amostras. O espectro de massas de alta resolução foi obtido em espectrômetro utilizando a técnica ESI (MicrOTOF II - ESI TOF, Bruker-daltons, MA, USA). Cromatografias em coluna aberta foram realizadas utilizando gel de sílica 60 (0,040-0,063 $\mathrm{mm}$ e 0,063-0,200 mm, ambas da merck). Para cromatografia em camada delgada comparativa (CCDC) foram utilizadas cromatoplacas em fase normal e fase reversa RP-18 recortadas $(5 \times 5 \mathrm{~cm})$ e as substâncias foram evidenciadas em CCDC pelo uso de radiaçáo ultravioleta (UV) sob comprimentos de onda de 254 e $366 \mathrm{~nm}$, pela impregnação das placas em cubas de vidro saturadas por vapores de iodo, soluçáo de anisaldeído seguido de aquecimento e soluçáo de Dragendorff. As análises foram efetuadas em cromatógrafo líquido semipreparativo (7725i, Shimadzu, Kyoto, Japan). A aquisição dos dados foi feita utilizando o programa CLASS-VP versão 6.13 SP2. Uma coluna semipreparativa SHIM-PACK PREP-ODS H (250 $\mathrm{mm} \times 20 \mathrm{~mm}$ de $5 \mu \mathrm{m}$ ) foi utilizada.

O material botânico (galhos e cascas do tronco) foi coletado na Reserva Adolpho Ducke, em outubro de 2007, no $\mathrm{km} 26$ da rodovia Manaus-Itacoatiara a partir de espécimes previamente identificados no Projeto Flora da Reserva Ducke. A exsicata de número 189608 foi atribuída ao espécime de
Parahancornia amapa (Huber) coletado e está depositada no herbário do Instituto Nacional de Pesquisa da Amazônia.

Os galhos de P. amapa $(700 \mathrm{~g})$ secos e moídos foram submetidos à extraçáo em aparelho tipo Soxhlet $(3 \times 400 \mathrm{~mL}$, $3 \times 6$ horas) com hexano e posteriormente com diclorometano. Após filtração e evaporação sob pressão reduzida, foi obtido 4,3 $\mathrm{g}$ do extrato diclorometânico dos galhos. Parte deste extrato $(750 \mathrm{mg})$ foi submetida à cromatografia em coluna $(\mathrm{h}=45$ $\mathrm{cm} ; \varnothing=4,0 \mathrm{~cm})$ de sílica gel eluída com a mistura de hexano/ DCM (70:30), DCM (100) e DCM/MeOH (90:10, 80:20, 70:30) resultando 72 fraçóes, as quais foram reunidas em sete grupos (A1-A7). A fração A1 (61,0 mg) foi submetida à separação cromatográfica em coluna $(\mathrm{h}=15 \mathrm{~cm} ; \varnothing=2,0 \mathrm{~cm})$ de sílica gel $60(0,063-0,200 \mathrm{~mm})$ eluída em gradientes de hexano:DCM (80:20), hexano:DCM (50:50) e DCM (100) resultando 20 fraçóes, as quais foram reunidas em três grupos (B1-B3). A fração B1 (12,7 mg) forneceu um sólido cristalino branco identificado como a mistura de esteroides estigmasterol (amostra um) e $\beta$-sitosterol (amostra dois). As fraçóes A2 (90,0 mg) e A6 (256,0 mg), após o fracionamento em CCDP utilizando-se como eluentes misturas de hexano/AcOEt (7:3), DCM/AcOEt (9:1) forneceram, respectivamente, friedelina (amostra três) (18,0 mg) e uma mistura $(12,3 \mathrm{mg})$ composta por $\alpha$-amirina (amostra 4) , $\beta$-amirina (amostra cinco) e lupeol (amostra seis) em forma de um sólido amorfo branco (Figura 1). As cascas do tronco de P. amapa (400 g) secas e moídas foram submetidas à extração em aparelho tipo Soxhlet (3x $400 \mathrm{~mL}, 3 \times 6$ horas) com aumento de polaridade utilizando hexano, DCM, AcOEt e posteriormente metanol. Após filtração e evaporação sob pressão reduzida, foi obtido $18,9 \mathrm{~g}$ do extrato metanólico das cascas. Esse foi particionado com solventes de polaridade crescente, obtendo-se as fraçốes hexânica (FH), diclorometânica (FD), acetato de etila (FAc) e metanólica (FM). A fração FD $(2,2 \mathrm{~g})$ foi submetida à separação cromatográfica em coluna $(h=35 \mathrm{~cm} ; \varnothing=3,0$ $\mathrm{cm}$ ) de sílica gel 60 eluída em mistura de $\mathrm{Me}_{2} \mathrm{CO} / \mathrm{MeOH}$ até $\mathrm{MeOH}(100 \%)$ resultando 94 fraçóes, as quais foram reunidas em 14 grupos (FD1-FD14). Posteriormente, a fração FD10 $(480,0 \mathrm{mg})$ foi purificada em cromatografia líquido de alta eficiência (CLAE) semipreparativo, utilizando como fase móvel a mistura dos solventes $\mathrm{MeOH} / \mathrm{H}_{2} \mathrm{O}$ (30:70), em condição isocrática, com fluxo de $14 \mathrm{~mL} / \mathrm{min}$. Para a detecção dos picos de interesse foi utilizado o comprimento de onda de $254 \mathrm{~nm}$ para o alcaloide isositsiriquina (amostra sete) (Figura 1) $(t r=18,2$ minutos; $16,2 \mathrm{mg})$.

O estudo fitoquímico dos galhos de $P$. amapa resultou na identificação da mistura dos esteroides estigmasterol (amostra um) e $\beta$-sitosterol (amostra dois) foram identificadas pela análise dos espectros de Ressonância Magnética Nuclear (RMN) de ${ }^{1} \mathrm{H}$ e ${ }^{13} \mathrm{C}$ e comparação com dados da literatura (Ahmed et al. 1992; Goulart et al. 1993). 


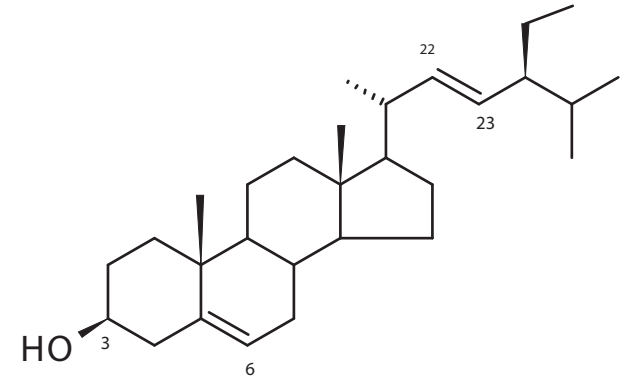

(1)
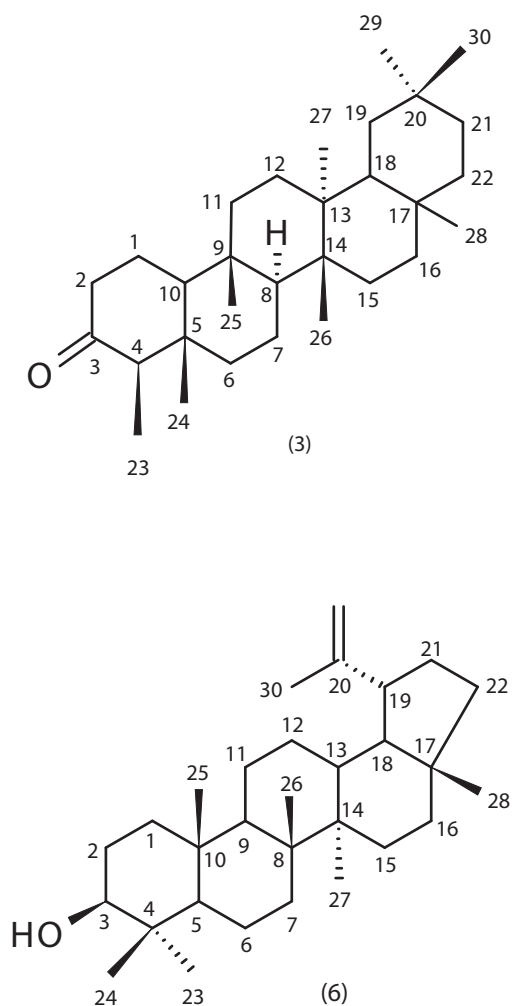<smiles>CCC(CC[C@@H](C)C1CCC2C3CC=C4C[C@@H](O)CC[C@]4(C)C3CCC21C)C(C)C</smiles>

(2)

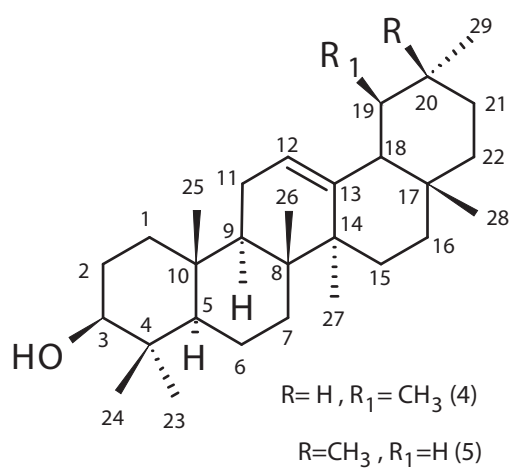

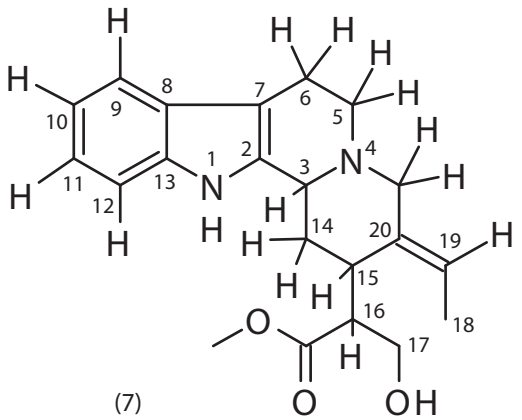

Figura 1. Estruturas químicas dos esteroides (amostra um e dois), triterpenos pentacíclicos (amostras três, quatro, cinco e seis) e do alcaloide (amostra sete) isolados de $P$. amapa.

Pela análise do espectro de Ressonância Magnética Nuclear (RMN) de ${ }^{1} \mathrm{H}$ da amostra três foram observados sinais de hidrogênio de sete metilas em simpletos $\left(\delta_{\mathrm{H}} 1,18\right.$, $1,05,1,01,1,00,0,95,0,87$ e 0,73 ) além de um dupleto em $\delta 0,88(J 7,0 \mathrm{~Hz})$, referente ao grupo metila $\mathrm{H}-23$ que é característico de esqueleto friedelano. Pela análise dos espectros de Ressonância Magnética Nuclear (RMN) de ${ }^{13} \mathrm{C}$ e de intensificação sem distorçáo por transferência de polarização (DEPT) foram observadas a presença de um sinal em $\delta 213,3$, referente ao carbono da carbonila e um sinal em $\delta$ 7,0 indicando a presença de um grupo metílico (C-23), esses valores sugerem a presença de um triterpeno da série friedelano (Akihisa et al . 1992; Mahato 1994; Abreu et al. 2013). A análise dos dados espectrais e a comparação com os dados da literatura disponíveis permitiram caracterizar a amostra três como friedelina. 
$\mathrm{Na}$ análise do espectro de Ressonância Magnética Nuclear (RMN) de ${ }^{1} \mathrm{H}$ para a mistura de triterpenos (amostras quatro, cinco e seis) foram observados os sinais de simpletos entre $\delta$ 0,8 e $\delta$ 1,2 característicos de hidrogênios de esqueletos triterpênicos, dois tripletos largos com integração para um hidrogênio cada, em $\delta 5,18$ e $\delta 5,13$, atribuídos a hidrogênios ligados a carbonos olefínicos de esqueleto ursano e oleano, respectivamente (Mahato et al. 1994). Os sinais de dupleto largo e duplo dupleto com integraçáo para um hidrogênio cada, acoplando entre si, em $\delta$ 4,69 e 4,57 caracterizam os hidrogênios vinílicos presentes na amostra seis (Mahato et al. 1994; Costa et al. 2010). O espectro de Ressonância Magnética Nuclear (RMN) de ${ }^{13} \mathrm{C}$ e de intensificação sem distorção por transferência de polarização (DEPT) (125 $\mathrm{MHz}, \mathrm{CDCl}_{3}$ ) apresentaram sinais que permitiram sugerir seu esqueleto triterpênico em: $\delta$ 139,1 (C-13) e $\delta$ 124,3 (C-12), em $\delta 145,1(\mathrm{C}-13)$ e $\delta 121,5$ (C-12) e em: $\delta 150,9$ (C-20) e $\delta 109,5$ (C-29) atribuídos a carbonos insaturados tipo $\mathrm{sp}^{2}$ que são compatíveis com triterpenos pentacíclicos das séries ursano, oleanano e lupano, respectivamente (Mahato et al. 1994; Cursino et al. 2009). Foram observados sinais em $\delta$ 79,0 para os carbonos carbinólicos C-3 e em $\delta$ 19,5 sugerido a um grupo metila (C-30) ligado a carbono $\mathrm{sp}^{2}$ da série lupano (Olea et al.1990; Mahato et al. 1994). Consequentemente com base nos resultados das análises dos dados espectrais e a comparação com os dados da literatura a mistura foi identificada como $\alpha$-amirina (amostra 4), $\beta$-amirina (amostra 5) e lupeol (amostra 6), respectivamente. O isolamento e identificação desses constituintes revelaram a semelhança com os registrados na literatura para estudos químicos anteriores com o gênero Parahancornia.

A amostra sete apresentou-se como uma resina amarela, teste positivo para alcaloides frente ao reagente de Dragendorff e fórmula molecular $\mathrm{C}_{21} \mathrm{H}_{27} \mathrm{~N}_{2} \mathrm{O}_{3}$ deduzida do seu espectro de massas de alta resoluçấo $[\mathrm{M}+\mathrm{H}]^{+}$355,2012 (calculado 355,2016). O espectro de Ressonância Magnética Nuclear (RMN) de ${ }^{1} \mathrm{H}$ da substância (amostra sete) apresentou quatro sinais correspondentes a hidrogênios aromáticos em $\delta 7,49$ (d, 8,0 Hz, H-9), $\delta 7,07$ (t, 8,0 Hz, H-10), $\delta 7,18$ (t, 8,0 Hz, $\mathrm{H}-11), \delta 7,44(\mathrm{~d}, 8,0 \mathrm{~Hz}, \mathrm{H}-12)$, que sugeriram a natureza indólica da substância (Tabela 1). A análise do espectro de ${ }^{13} \mathrm{C}$ permitiu identificar 21 sinais (Tabela 1), que em conjunto com o mapa de contornos de correlação heteronuclear de um único quantum (HSQC), espectro de intensificação sem distorção por transferência de polarizaçáo (DEPT) e o espectro de Ressonância Magnética Nuclear (RMN) de ${ }^{1} \mathrm{H}$ evidenciaram a presença de quatro carbonos metínicos com $\delta$ 119,34 (C9), $\delta 120,77$ (C-10), $\delta 123,84$ (C-11) e $\delta 112,78$ (C-12) correlacionando com os hidrogênios em $\delta 7,49(\mathrm{~d}, 8,0 \mathrm{~Hz}$,

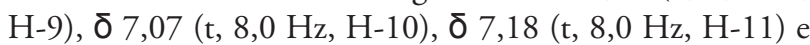
$\delta 7,44(\mathrm{~d}, 8,0 \mathrm{~Hz}, \mathrm{H}-12)$, respectivamente. Também foi possível verificar a presença de um simpleto em $\delta$ 3,03
$(3 \mathrm{H}, s)$ atribuído à metila da metoxila e em $\delta 1,88(3 \mathrm{H}, \mathrm{d}$, $\mathrm{J} 6,5 \mathrm{~Hz}$ ) referente à metila do grupo etilidênico. Para esse grupo etilidênico também foi observada a presença de um quarteto atribuído ao hidrogênio olefínico em $\delta 6,05$ (q, 6,5 $\mathrm{Hz}, \mathrm{H}-19)$. O mapa de contornos de correlaçấo heteronuclear de múltiplos quanta (HMBC) possibilitou determinar as seguintes correlaçóes entre o sinal em $\delta 1,88(3 \mathrm{H}-18)$ com o (C-20) em $\delta 129,5$; o sinal em $\delta 6,05$ (H-19) com o (C-21) em $\delta$ 68,34; entre os sinais dos $\mathrm{H}-15(\delta 3,37)$ e $\mathrm{H}-21 \mathrm{a}(\delta 3,70)$ com C-19 $\delta 135,46$ que permitiram observar a conexáo entre a cadeia olefínica e o núcleo indolquinolizidínico. Também foram observadas as correlaçóes entre os sinais dos hidrogênios em $\delta 3,37$ (H-15), ठ 3,73 (H-17b), ठ 3,63 (H-17a) e ठ 2,47 (H-16) com o sinal $\delta 180,40(\mathrm{C}=\mathrm{O})$, atribuído a carbonila do grupo éster. A análise do espectro de efeito nuclear Overhauser (NOESY) mostrou as correlaçóes dos hidrogênios em $\delta$ 1,88 (3H-18) com os sinais dos hidrogênios em $\delta$ 3,36

Tabela 1. Dados de RMN (500 MHz, $\left.\mathrm{CD}_{3} \mathrm{OD}\right)$ de ${ }^{1} \mathrm{H}$ e ${ }^{13} \mathrm{C}$ do alcaloide isositsiriquina (amostra sete) isolado de $P$. amapa.

\begin{tabular}{|c|c|c|}
\hline $\mathrm{N}^{0} \mathrm{H} / \mathrm{C}$ & $\delta \mathrm{H}$, (multip., J Hz) & $\delta C$ \\
\hline 2 & - & 128,65 \\
\hline 3 & 4,33 (1H, d, $11 \mathrm{~Hz})$ & 66,54 \\
\hline $5 a$ & $3,36(\mathrm{~m}, 1 \mathrm{H})$ & 59,07 \\
\hline $5 b$ & $3,54(\mathrm{~m}, 1 \mathrm{H})$ & \\
\hline 6 & $2,97(\mathrm{~m}, 2 \mathrm{H})$ & 18,24 \\
\hline 7 & - & 105,68 \\
\hline 8 & - & 126,99 \\
\hline 9 & $7,49(\mathrm{~d}, 8,0 \mathrm{~Hz}, 1 \mathrm{H})$ & 119,34 \\
\hline 10 & $7,07(\mathrm{t}, 8,0 \mathrm{~Hz}, 1 \mathrm{H})$ & 120,77 \\
\hline 11 & 7,18 (t, 8,0 Hz, 1H) & 123,84 \\
\hline 12 & $7,44(\mathrm{~d}, 8,0 \mathrm{~Hz}, 1 \mathrm{H})$ & 112,78 \\
\hline 13 & - & 138,75 \\
\hline $14 a$ & $2,33(\mathrm{~m}, 1 \mathrm{H})$ & 30,48 \\
\hline $14 b$ & $2,51(\mathrm{~m}, 1 \mathrm{H})$ & \\
\hline 15 & $3,37(\mathrm{~m}, 1 \mathrm{H})$ & 35,64 \\
\hline 16 & $2,47(\mathrm{~m}, 1 \mathrm{H})$ & 54,47 \\
\hline $17 a$ & $3,63(\mathrm{~m}, 1 \mathrm{H})$ & 62,85 \\
\hline $17 \mathrm{~b}$ & $3,73(\mathrm{~m}, 1 \mathrm{H})$ & \\
\hline 18 & $1,88(\mathrm{~d}, 6,5 \mathrm{~Hz}, 3 \mathrm{H})$ & 14,38 \\
\hline 19 & $6,05(q, 6,5 \mathrm{~Hz}, 1 \mathrm{H})$ & 135,46 \\
\hline 20 & - & 129,52 \\
\hline $21 \mathrm{a}$ & $3,70(\mathrm{~d}, 13,0 \mathrm{~Hz}, 1 \mathrm{H})$ & 68,34 \\
\hline $21 b$ & $4,19(\mathrm{~d}, 13,0 \mathrm{~Hz}, 1 \mathrm{H})$ & \\
\hline 22 & - & 180,40 \\
\hline $\mathrm{OCH}_{3}$ & $3,03(\mathrm{~s}, 3 \mathrm{H})$ & 48,42 \\
\hline
\end{tabular}


(H-5a), ठ 2,51 (H-14b) e $\delta$ 2,47 (H-16). Outras correlaçóes foram observadas pela análise do espectro de efeito nuclear Overhauser (NOESY) entre o sinal do hidrogênio em $\delta$ 3,37 (H-15) com $\delta 4,33$ (H-3) e entre o sinal dos hidrogênios $\delta 3,03(3 \mathrm{H}, \mathrm{s})$ com os sinais em $\delta 4,33(\mathrm{H}-3), \delta 3,70(\mathrm{H}-21 \mathrm{a})$ e $\delta$ 4,33 (H-3), sugerindo a orientação espacial desses átomos na estrutura.

A comparação com os valores publicados para o alcaloide isositsiriquina, bem como os dados obtidos pelo experimento de efeito nuclear Overhauser (NOESY), possibilitou sugerir a estereoquímica do composto, determinando a para H-3 $(S)$ e $\mathrm{H}-15(R)$ e $\beta$ para H-16 (R) e configuraçáo para a dupla ligação C19-C20 foi determinada como $E$, pois de acordo com trabalho anterior (Lounasmaa et al. 1994) essa configuração confere deslocamento $-\delta 33,0$ ppm ao carbono C-15 e para a configuração $Z$ atribui o valor de $-\delta 41,0 \mathrm{ppm}$. Com base nesses dados de Ressonância Magnética Nuclear (RMN) e a comparaçáo com os dados da literatura foi possível inferir que a amostra sete tratava-se do alcaloide indólico $(3 S, 15 R$, $16 R)$ - $E$-isositsiriquina.

Neste estudo foi confirmado à presença de triterpenos e esteroides característicos dessa espécie e em acréscimo ao conhecimento fitoquímico foi isolado o alcaloide indólico isositsiriquina. Este é o primeiro relato dessa classe química para o gênero Parahancornia, apesar de pertencer à família Apocynaceae, a qual é conhecida como bioprodutora dessa classe química. Classe essa que possui amplo espectro de atividades biológicas. Portanto esse estudo contribui com a determinação de novos constituintes químicos de $P$. amapa, a qual é bastante utilizada na Amazônia por suas propriedades medicinais.

\section{AGRADECIMENTOS}

À Fundaçáo de Amparo à Pesquisa do Estado do Amazonas (FAPEAM), pela bolsa de Doutorado concedida. Ao Centro de Biotecnologia da Amazônia na pessoa do Prof. Dr. Massayoshi Yoshida, pela obtenção dos espectros de Ressonância Magnética Nuclear (RMN) e ao Prof. Dr. N. P. Lopes (FCF/USP-RP) pela aquisição dos espectros de massas de alta resoluçáo.

\section{BIBLIOGRAFIA CITADA}

Abreu, V. G. da C.; Corrêa, G. M.; Lagos, I. A. dos S.; Silva, R. R. S.; Alcântara, A. F. de C. 2013. Triterpenos pentacíclicos e esteroides da casca do uchi (Sacoglottis uchi, Humiriaceae). Acta Amazonica, 43: 525 - 528.

Akihisa, T.; Yamamoto, K. Tamura, T.; Kimura, Y.; Iida, T.; Nambara, T.; Chang, F. C. 1992. Triterpenoid ketones from Lingnania chungii Mcclure-arborinone, friedelin and glutinone. Chemical and Pharmaceutical Bulletin, 40:789-91.

Ahmed, W.; Ahmad, Z.; Malik, A. 1992. Stigmasterol galactoside from Rhynchosia minima. Phytochemistry, 31: 4038-4039.

Carvalho, M. G.; Velloso, C. R. X.; Braz-Filho, R.; Costa, W. F. 2001. Acyl-lupeol esters from Parahancornia amapa (Apocynaceae). Journal of Brazilian Chemical Society, 12: 556-559.

Costa, D. A.; Chaves, M. H.; Silva, W. C. S.; Costa, C. L. S. 2010. Constituintes químicos, fenóis totais e atividade antioxidante de Sterculia striata St. Hil. et Naudin. Acta Amazonica, 40: 207-212.

Cursino, L. M. de C.; Mesquita, A. S.S.; Mesquita, D.W. de O.; Fernandes, C.C.; Pereira Junior, O.L.; Amaral, I.L.; Nunez, C.V. 2009. Triterpenos das folhas de Minquartia guianensis Aubl. (Olacaceae) Acta Amazonica, 39: 181-186.

Goulart, M. O. F.; Sant'Ana, A. E. G.; Lima, R. A.; Cavalcante, S. H.; Carvalho, M. G.; Braz-Filho, R. 1993. Fitoconstituintes químicos isolados de Jatropha elliptica. Atribuição dos deslocamentos químicos dos átomos de carbono e hidrogênio dos diterpenos jatrofolonas A e B. Química Nova, 16: 95-100.

Lounasmaa, M.; Jokew, R.; Hanbinen, P.; Mlettinen, J.; Sale, J. 1994. Preparation and conformational study of $Z$ - and $E$ isositsirikine epimers and model compounds. Determination of their C-16 configurations. Tetrahedron, 50, 9207- 9222.

Mahato, S. B., Kundu, A. P. $1994 .{ }^{13} \mathrm{C}$ NMR spectra of pentacyclic triterpenoids - a compilation and some salient features. Phytochemistry, 37: 1517-1575.

Mendonça, P. M.; Lima, M. G.; Albuquerque, L. A. O.; Carvalho, M. G.; Queiroz, M. C. 2010. Effects of Latex from "Amapazeiro" Parahancornia amapa (Apocynaceae) on blowfly Chrysomya megacephala (Diptera:Calliphoridae) post-embryonic development. Veterinary Parasitology, 178: 379-382.

Olea, R. S. G.; Roque, N. F. 1990. Análise de misturas de triterpenos por RMN ${ }^{13}$ C. Quimica Nova, 13: 278-281.

Sobrinho, D. C.; Hauptli, M. B.; Appolinário, E. V.; Kollenz, C. L. M.; Carvalho, M. G. de; Braz-Filho, R. 1991. Journal of Brazilian Chemical Society, 2: 15-20.

Recebido em 26/10/2013

Aceito em 14/03/2014 
